\title{
基于多目标粒子群算法的电磁主动悬架作动器优化
}

\author{
杨 超 ${ }^{1}$ 李以农 ${ }^{1,2}$ 郑 玲 $^{1}$ 胡一明 ${ }^{1}$ \\ (1. 重庆大学汽车工程学院 重庆 400030 ; \\ 2. 重庆大学机械传动国家重点实验室 重庆 400030)
}

\begin{abstract}
摘要 : 针对电磁主动悬架直线式作动器电磁力波动对悬架系统影响问题, 建立作动器磁场解析模型, 以总谐波畸变量(Total harmonic distortion, THD)作为电动势(Electromotive force，EMF)中谐波含量的评价指标，对影响电磁力输出的 EMF 进行谐 波分析，在此基础上，建立考虑悬架电磁力波动特性的悬架系统模型，分析了车辆动力学响应特性。其次，采用多目标粒子 群智能优化算法，以“大 EMF 幅值”和“ 小 THD”值作为目标，对作动器结构参数进行多目标优化，并利用模糊集合理论 对优化后的 Pareto 最优解集进行选优。仿真结果表明，作动器电磁力波动下降了 $53.8 \%$, 有效电磁力提升了 $8.5 \%$, 基本消除 了电磁力波动对悬架系统的影响。最后，对作动器样件进行测试，结果显示：作动器绕组 EMF 中含有 3 次、2 次、4 次和 5 次谐波分量，且 THD 值达到了 $5.6 \%$, 电磁力波动为 $7.8 \mathrm{~N}$, 试验结果验证了对电磁力波动分析及优化的有效性。

关键词 : 电磁主动悬架 ; 直线作动器 ; 电磁力波动 ; 多目标优化 ; 粒子群算法
\end{abstract}

中图分类号 : TH122；U463

\section{Optimum of Electromagnetic Active Suspension Actuator Using Multi-objective Particle Swarm Optimization Algorithm}

\author{
YANG Chao $^{1}$ LI Yinong ${ }^{1,2}$ ZHENG Ling $^{1}$ HU Yiming $^{1}$
}

(1. College of Automotive Engineering, Chongqing University, Chongqing 400030;

2. State Key Laboratory of Mechanical Transmission, Chongqing University, Chongqing 400030)

\begin{abstract}
For aiming at reducing the influence of the thrust ripple in electromagnetic active suspension, magnetic field theoretical model of the actuator is established, total harmonic distortion (THD) is taken as the evaluation to actuator EMF, harmonic components of EMF which effective to the electromagnetic force are analyzed. On this basis, a suspension dynamics model considering suspension electromagnetic force fluctuation is established, and the vehicle dynamic response characteristics are analyzed. And then, taking the maximum EMF amplitude and the minimum THD as optimization objective, the actuator structure parameters are optimized with intelligent multi-objectives particle swarm optimization. The best Pareto optimal solution is selected based on the fuzzy set theory. After optimization, the ripple of electromagnetic force is reduced by $53.8 \%$, and the value of electromagnetic force is increased by $8.5 \%$, the influence of the thrust ripple in electromagnetic active suspension system is basically eliminated. Finally, actuator prototype is experimented on test bench. The results show that there are a series of harmonics including the 3rd, 2rd, 4rd and 5rd in the EMF wave, the THD is 5.6\%, and the ripple of electromagnetic force is $7.8 \mathrm{~N}$. Optimization results are verified by experiment.
\end{abstract}

Key words : electromagnetic active suspension ; linear actuator ; electromagnetic force fluctuation ; multi-objective optimization ; particle swarm algorithm

\section{0 前言}

传统的悬架系统不能够兼顾车辆的平顺性和操

* 国家重点研发计划(2017YFB0102603-4)和重庆市基础与前沿研究计划 (cstc2018jcyjAX0630)资助项目。20181006 收到初稿，20190302 收到修 改稿
稳性的平衡，电磁主动悬架能够产生可调的主动控 制力, 进而从根本上解决这一矛盾 ${ }^{[1-3]}$ 。目前作为电 磁主动悬架核心部件之一的电磁作动器鲜有研究, 而作动器性能的优劣将直接影响电磁主动悬架的发 展及在智能电动车辆上的应用。

现阶段, 针对作动器的研究主要分为旋转式作 动器 ${ }^{[4-6]}$ 和直线式作动器 ${ }^{[7-9]}$, 其中旋转式作动器其 
传动链较长, 效率较低, 而且动力传递机构中齿轮 的啮合容易出现松动, 进而降低系统的稳定性。直 线式作动器省略了动力传递机构，结构更为紧凑， 传递效率更高。文献[10-12]利用电磁感应原理分别设 计了感应式作动器、磁阻式作动器和永磁式作动器。 但此类直线作动器均忽略了齿槽结构、端部效应以 及磁场谐波带来的电磁力波动的问题, 直接影响电 磁力精确输出, 从而给悬架控制器的设计带来困难。

基于上述分析，针对齿槽结构产生的齿槽效应， 文献[13]采用分数槽的形式设计电磁悬架作动器, 从最优极槽匹配的角度降低电磁力的波动;文献[14] 则根据齿槽力产生的原理，对齿槽和永磁体的形状 进行改进，运用斜槽和斜极的结构降低了电磁力的 波动。文献[15]考虑作动器的端部效应, 从端部力 着手优化动子长度, 从而降低了电磁力的波动, 但 该方法在削弱电磁力波动的同时, 也造成了有效电 磁力输出的降低。

但以往的研究大多基于电磁作动器本体, 对作 动器和悬架系统的特性分析较少, 不能较好地反应 作动器特性对悬架系统的影响, 因此, 本文从磁场 谐波的角度(主要为感应电动势谐波)对电磁主动悬 架直线式作动器进行电磁力波动分析, 在此基础上 建立了考虑电磁力波动的主动悬架动力学模型, 分 析了作动器电磁力波动对主动悬架性能的影响, 针 对这种影响, 从作动器本体设计角度, 利用多目标 粒子群智能优化算法, 对电磁主动悬架直线式作动 器进行结构参数的优化, 降低电磁力波动以及提高 有效电磁力输出, 以消除作动器电磁力波动对主动 悬架性能的影响, 最后通过作动器台架试验对作动 器的优化设计进行了验证。

\section{1 电磁主动悬架作动器}

\section{1 馈能悬架作动器结构}

图 1 为电磁主动悬架作动器结构示意图, 其中 作动器初级铁心(定子)与轮轴、轮毂、电机和轮胎 等簧下质量相连; 次级铁心(动子)与车架等簧上质 量相连, 初级和次级铁心在电流激励下产生相对运 动和电磁力。此外, 被动工作时初级铁心绕组在次 级磁场中产生感应电动势, 通过馈能电路向蓄电池 充电, 能够实现振动能量回收。

本文作动器采用圆筒形设计, 结合作动器的长 度, 为降低作动器齿槽力以及提高作动器绕组系数, 选用槽数为 12 极数为 14 的分数槽结构 ${ }^{[15]}$ 。作动器 定子和动子采用导磁良好的 10 号钢, 永磁体材料为 钕铁硼 $\mathrm{N} 30$, 绕组线圈采用直径为 $1 \mathrm{~mm}$ 的铜线。

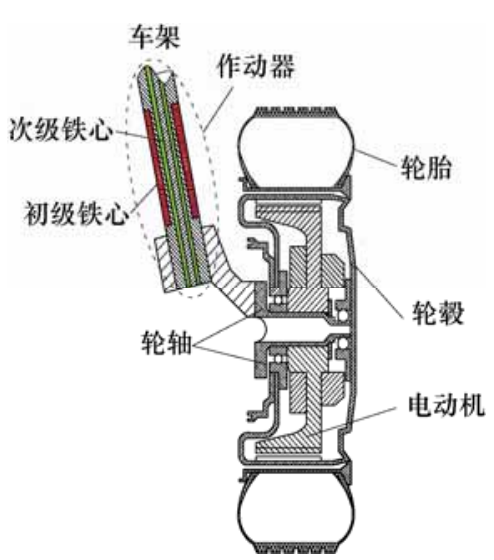

图 1 直线式馈能悬架作动器示意图

作动器具体结构尺寸如图 2 所示, 具体参数见 表 1 。

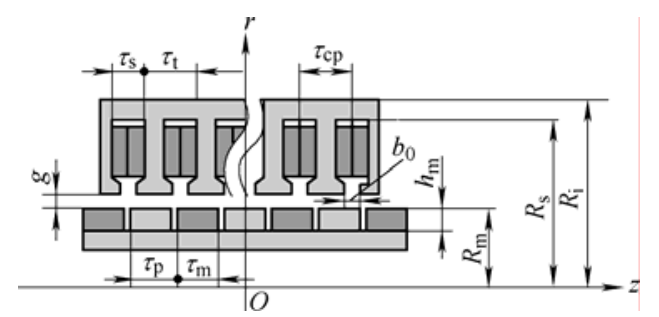

图 2 作动器结构尺寸

表 1 作动器结构参数

\begin{tabular}{lc}
\hline \multicolumn{1}{c}{ 参数 } & 12 级 $/ 14$ 槽 \\
\hline 槽口宽 $b_{0} / \mathrm{mm}$ & 3 \\
槽距 $\tau_{\mathrm{t}} / \mathrm{mm}$ & 14 \\
极距 $\tau_{\mathrm{p}} / \mathrm{mm}$ & 12 \\
永磁体长度 $\tau_{\mathrm{m}} / \mathrm{mm}$ & 10 \\
极弧系数 & 0.8 \\
槽宽 $\tau_{\mathrm{s}} / \mathrm{mm}$ & 7 \\
永磁体厚度 $b_{m} / \mathrm{mm}$ & 3 \\
每槽匝数 $N$ & 100 \\
初级外半径 $R_{\mathrm{i}} / \mathrm{mm}$ & 40 \\
次级外半径 $R_{\mathrm{m}} / \mathrm{mm}$ & 20 \\
定子长度 $L / \mathrm{mm}$ & 168 \\
电枢半径 $R_{\mathrm{s}} / \mathrm{mm}$ & 35 \\
气隙长度 $g / \mathrm{mm}$ & 2 \\
\hline
\end{tabular}

\section{2 模型的建立及验证}

\section{1 感应电动势解析}

感应电动势是衡量电磁作动器电磁力输出的 主要参数之一。为分析本文作动器性能, 需要建立 感应电动势解析模型。参考文献[16]，圆筒形永磁 直线作动器气隙绕组磁链对时间求导, 可求得感应 电动势

$$
E=\frac{\mathrm{d} \Phi}{\mathrm{d} t}=-v \sum_{n=1,2, \cdots}^{\infty} K_{\mathrm{E} n} \sin m_{n}\left(z-\frac{\tau_{\mathrm{cp}}}{2}\right)
$$


式中 $E$ 一一感应电动势；

$\Phi$ - 磁链 ;

$v$ 一运行速度；

$K_{\mathrm{E} n}$ 一感应电动势系数 ;

$n$ 一谐波阶次 $n=1,2, \cdots$;

$m_{n}$ —磁链的角频率 ;

$z$ 一一移动距离 ;

$\tau_{\mathrm{cp}}$ 一线圈节距，本文槽距和节距相同。

$K_{\mathrm{E} n}$ 可以表示为

$$
K_{\mathrm{E} n}=4 \pi p N_{\mathrm{c}} K_{\mathrm{dp} n} K_{\mathrm{r} n}
$$

式中 $p$ 一极对数 ;

$N_{c}$ 每极每相线圈匝数 ;

$K_{\mathrm{dp} n}$ 一绕组系数 ;

$K_{\mathrm{r} n}$ 气隙磁场分布系数。

$K_{\mathrm{dpn} n}$ 可表示为

$$
K_{\mathrm{dp} n}=\frac{\sin \left(\frac{m_{n} b_{0}}{2}\right)}{\left(\frac{m_{n} b_{0}}{2}\right)}\left(\frac{m_{n} \tau_{\mathrm{cp}}}{2}\right)
$$

式中， $b_{0}$ 为槽口宽度，本文中 $b_{0}$ 即为槽宽 $\tau_{\mathrm{s}}$ 。

气隙磁场分布系数 $K_{\mathrm{rn}}$ 可表示为

$$
K_{\mathrm{r} n}=R_{\mathrm{se}}\left[a_{\mathrm{I} n} B I_{1}\left(m_{n} R_{\mathrm{se}}\right)+b_{\mathrm{I} n} B K_{1}\left(m_{n} R_{\mathrm{se}}\right)\right]
$$

式中， $a_{\mathrm{I} n}$ 和 $b_{\mathrm{I} n}$ 为一类贝塞尔函数 $B I_{1}(\bullet)$ 和 $B K_{1}(\bullet)$ 的 系数, 主要和作动器的极弧系数和有效电枢厚度 $R_{\mathrm{se}}$ 有关。

有效电枢厚度 $R_{\mathrm{se}}$ 可表示为

$$
R_{\mathrm{se}}=R_{\mathrm{m}}+g_{\mathrm{e}}
$$

式中 $R_{\mathrm{m}}$ 一永磁体外半径 ;

$g_{\mathrm{e}}$ 一气隙有效计算长度。

$g_{\mathrm{e}}$ 可由下式计算

$$
g_{\mathrm{e}}=g+\left(K_{\mathrm{c}}-1\right) g^{\prime}
$$

式中， $K_{\mathrm{c}}$ 为卡特系数; $g^{\prime}$ 为考虑了齿槽效应的作 动器气隙长度, 两者分别表示为

$$
\begin{gathered}
K_{\mathrm{c}}=\frac{\tau_{\mathrm{t}}}{\tau_{\mathrm{t}}-\gamma g^{\prime}} \\
g^{\prime}=g+\frac{h_{\mathrm{m}}}{\mu_{\mathrm{r}}}
\end{gathered}
$$

式中 $\tau_{\mathrm{t}}$ 一槽距 ;

$\mu_{\mathrm{r}}$ ——相对磁导率，取 1.04 ;

$h_{\mathrm{m}}$ 一永磁体厚度。

齿槽系数 $\gamma$ 可表示为

$$
\gamma=\frac{4}{\pi}\left\{\frac{b_{0}}{2 g^{\prime}} \arctan \left(\frac{b_{0}}{2 g^{\prime}}\right)-\ln \sqrt{1+\left(\frac{b_{0}}{2 g^{\prime}}\right)^{2}}\right\}
$$

\section{2 作动器电磁力分析}

相比于传统减振器减振力特性, 电磁悬架电磁 阻尼力可以随电流和速度的大小进行实时调节。当 通入电流激励时，作动器电磁力可以表示为

$$
F=\frac{P}{v}=\frac{E_{A} \cdot I_{A}+E_{B} \cdot I_{B}+E_{C} \cdot I_{C}}{v}
$$

其中 $P$ 为作动器输入功率 $; E_{A} 、 E_{B} 、 E_{C}$ 分别为三 相感应电动势; $I_{A} 、 I_{B} 、 I_{C}$ 分别为三相电流 $; v$ 为 作动器工作速度。

根据式(1)感应电动势的解析, 设定作动器计算 的定工况, 定工况速度为 $0.24 \mathrm{~m} / \mathrm{s}$, 峰值电流为 $2 \mathrm{~A}$, 相对运行距离为 $150 \mathrm{~mm}$, 输入三相交流电

A 相

$$
I_{A \max } \sin (\omega t-2 \pi / 3+\varphi)
$$

$\mathrm{B}$ 相

$$
I_{B \max } \sin (\omega t+\varphi)
$$

$\mathrm{C}$ 相

$$
I_{C \max } \sin (\omega t+2 \pi / 3+\varphi)
$$

式中， $I_{\text {max }}$ 为各相电流峰值，电流角频率 $\omega=2 \pi f$; $f$ 为电流的频率 ; $\varphi$ 为初相位角，和感应电动势初 始相位一致。

感应电动势(单相)和电磁力仿真结果分别如图 3 和图 4 所示。从图 3 中可以看出，作动器感应电 动势基本上呈正弦分布。从图 4 中看出, 作动器电 磁力输出并非是稳定的数值，存在一定周期性的波 动，最大值 $113.0 \mathrm{~N}$,最小值 $105.2 \mathrm{~N}$, 波动为 $7.8 \mathrm{~N}$ 。

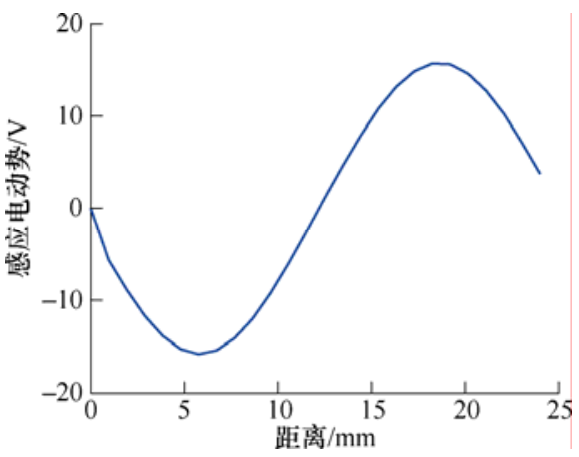

图 3 感应电动势曲线

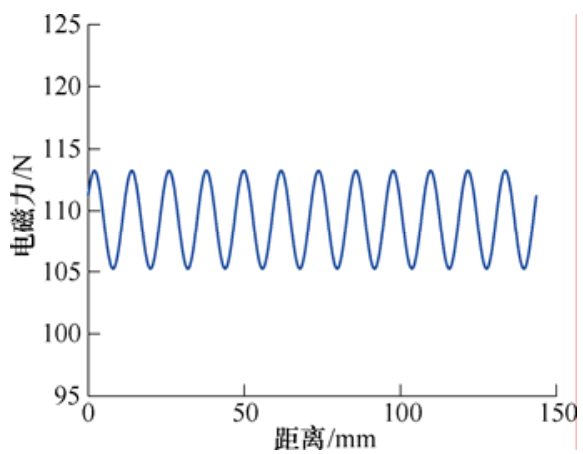

图 4 作动器电磁力 
这种波动会造成电磁力输出精度降低, 同时也会引 起系统的结构振动, 降低系统的可靠性, 因此必须 对其进行有效的抑制。

\section{3 感应电动势谐波分析}

在理想的三相正弦感应电动势和电流作用下作 动器作动力无电磁力波动，但从式(1)中可以看出， 作动器绕组感应电动势含有一系列谐波分量 , 在与 输入的三相电流作用下将造成电磁力波动。由于仿 真输入的电流激励为理想正弦波，因此本文对电磁 力波动的优化着重从作动器感应电动势所含谐波上 进行分析, 这里用各谐波所占基波比 $\eta$ 来评价各谐 波分量，表示为

$$
\eta=\frac{G_{n}}{G_{1}} \times 100 \%
$$

式中, $G_{n}$ 为第 $n$ 次谐波分量有效值; $G_{1}$ 为基波分量 有效值, 由于高次谐波所占基波比值较低, 这里 $n$ 取到 7 。

作动器的感应电动势的各谐波分量占比如图 5 所示。由图 5 可知, 除基波外感应电动势谐波主要 存在 3 次、 2 次、 4 次和 5 次谐波分量，其中 3 次谐 波分量所占基波比例最大, 为 $4.2 \%$ 。2 次谐波、 4 次谐波和 5 次谐波所占基波比分别为 $3.1 \% 、 1.9 \%$ 和 $1.3 \%$ 。

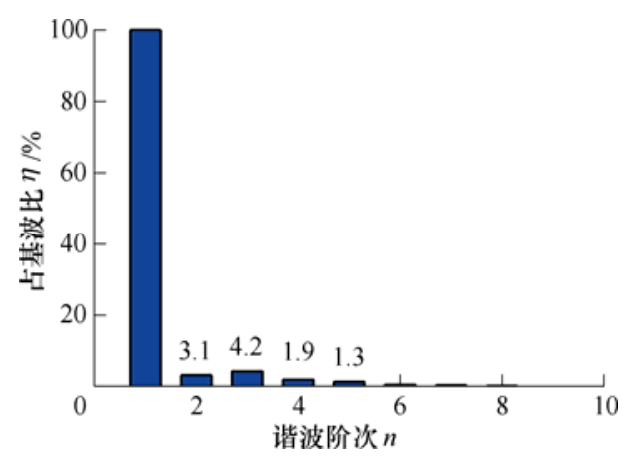

图 5 感应电动势谐波分量

以总谐波畸变率 THD 值对作动器感应电动势 总谐波含量进行评价，表示为

$$
\mathrm{THD}=\sqrt{\sum_{n=2}^{H}\left(\frac{G_{n}}{G_{1}}\right)^{2}} \times 100 \%
$$

式中 $H$ 指定阶次数。

经计算, 作动器感应电动势中 THD 值约为 $5.6 \%$ 。

结合感应电动势的解析式, 可以看出, 影响感 应电动势谐波含量的因素主要为作动器的结构参 数。当作动器长度、极数、槽数及绕组匝数一定的
情况下, 主要参数为永磁体的极弧系数 $\alpha_{\mathrm{p}}$ 、槽宽 $\tau_{\mathrm{s}}$ 、 永磁体厚度 $h_{\mathrm{m}}$ 、气隙 $g$ 。分析各参数对感应电动势 THD 值的影响，结果如图 6 所示。

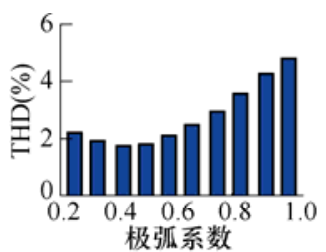

(a) 极弧系数

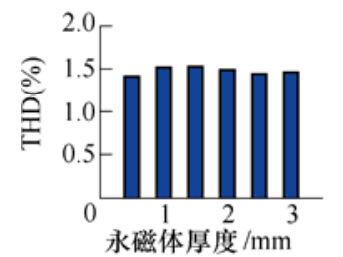

(c) 永磁体厚度

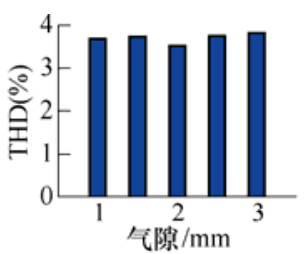

(b) 气隙长度

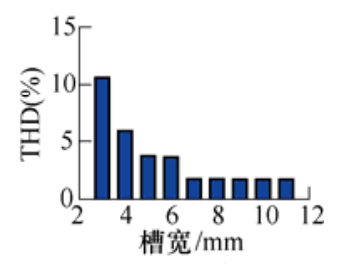

(d) 槽宽
图 6 各结构参数对感应电动势 THD 影响

从图 6 中可以看出, 极弧系数增加, THD 值 先降后升, 其中在 $0.4 \sim 0.5$ 时存在最小 THD 值; 随着气隙长度和永磁体厚度的增加, THD 值变化 较小, 即认为两者对感应电动势波形影响较小; 随 槽宽的增加，THD 值下降较快，当槽宽达到 $7 \mathrm{~mm}$ 时, THD 值基本保持平稳。此外, 各参数在改变 感应电动势 THD 值的同时, 也影响了感应电动势 的幅值 $E_{\mathrm{m}}$, 这里分析各参数对 $E_{\mathrm{m}}$ 的影响, 结果如 图 7 所示。

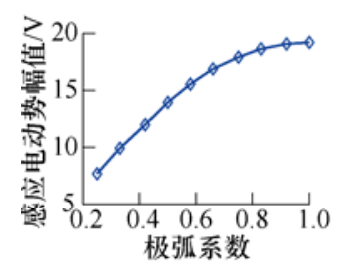

(a) 极弧系数

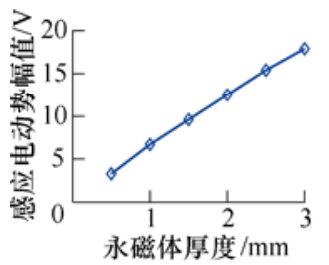

(c) 永磁体厚度

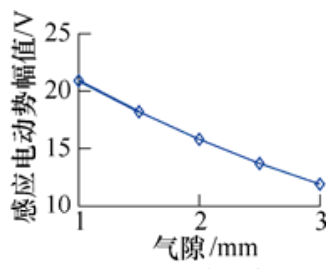

(b) 气隙长度

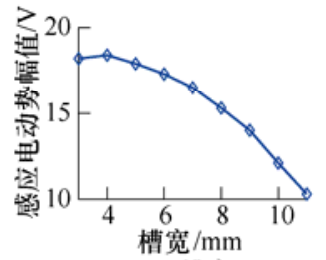

(d) 槽宽
图 7 各结构参数对感应电动势幅值 $E_{\mathrm{m}}$ 影响

从图 7 中可以看出: 随极弧系数和永磁体厚度 的增加，感应电动势幅值 $E_{\mathrm{m}}$ 呈增大趋势; 而随气 隙长度和齿槽宽度的增加 $E_{\mathrm{m}}$ 则呈逐渐降低。主要 是因为永磁体极弧系数和厚度的增加使得作动器 气隙处磁通增大, 进而增大了 $E_{\mathrm{m}}$ 。相反, 气隙和 槽宽的增加, 一方面降低了气隙的磁通密度, 另一 方面减小了经过齿部的有效磁通，从而造成 $E_{\mathrm{m}}$ 的 下降。 


\section{4 考虑电磁力波动的主动悬架特性}

\section{1 考虑电磁力波动的主动悬架动力学模型}

综上所述，作动器电磁力输出并非是稳定的 数值, 存在一定周期性的波动, 这种波动会造成 电磁力输出精度降低, 影响电磁主动悬架的动力 学性能及主动控制效果。本文建立含电磁力波动 的主动悬架动力学模型来分析电磁力波动对系统 的影响。

如图 8 所示为考虑电磁力波动的主动悬架动 力学模型, 模型由两部分组成, 含电磁力波动的作 动器输出模型与四分之一车辆动力学模型。其中 $M_{\mathrm{s}} 、 M_{\mathrm{u}}$ 为簧载质量和非簧载质量 $; K_{\mathrm{s}} 、 \mathrm{C}_{\mathrm{s}}$ 为悬架 的刚度和阻尼; $K_{\mathrm{t}}$ 为轮胎刚度; $Z_{\mathrm{s}} 、 Z_{\mathrm{u}} 、 Z_{\mathrm{g}}$ 分别为 簧载质量位移、非簧载质量位移以及路面位移激 励; $F_{\mathrm{a}}$ 为主动控制力， $K_{\mathrm{e}}$ 为不包含波动项的线性 电动机常数。

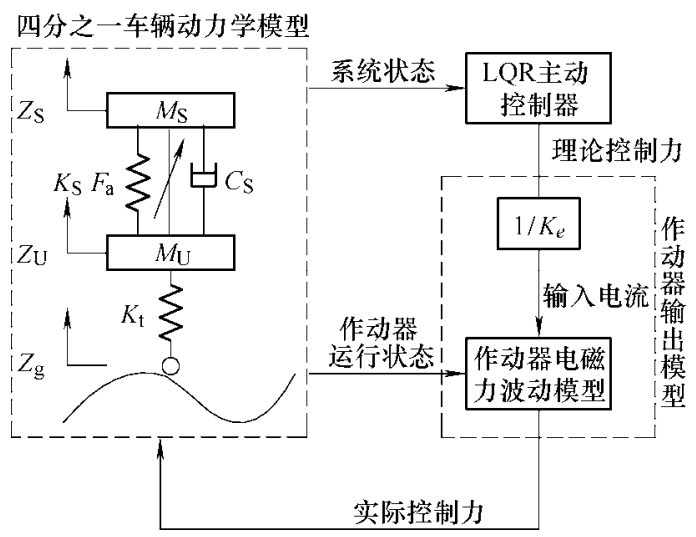

图 8 电磁力波动主动悬架系统框图

如图所示, 系统的仿真原理为, LQR 主动控制 器求出悬架系统需求的理论控制力, 再求出不含波 动力的作动器输入电流, 结合作动器的运行状态得 到含波动力的作动器实际输出电磁力。将实际输出 电磁力作为 $F_{\mathrm{a}}$ 对车辆系统施加主动控制力。

根据式(1)感应电动势的解析, 求作动器的波 动电磁力输出需要作动器的运行状态参数及输入 电流。作动器的运行状态参数包括作动器的运行 速度以及作动器的相对运行位置, 根据作动器在 悬架中的安装布局, 对应车辆动力学模型, 作动 器的运行速度为 $\dot{Z}_{u}-\dot{Z}_{s}$, 作动器的相对运行距离 为 $Z_{u}-Z_{s}$ 。

忽略谐波分量的电磁作动力表示为

$$
F=3 I_{m} K_{e}
$$

式中, $K_{\mathrm{e}}$ 为忽略谐波分量的线性电动机常数, 可通 过式(1)推导求得, $I_{\mathrm{m}}$ 为作动器输入电流, $F$ 为 $\mathrm{LQR}$
控制器输出的理论控制力。输入电流的最大值为峰 值电流 $I_{\max }$ 。

综上所述，根据输入电流 $I_{\mathrm{m}}$ 、作动器运行速度 $\dot{Z}_{u}-\dot{Z}_{s}$ 、作动器运行距离 $Z_{u}-Z_{s}$ 可求得包含电磁力 波动的作动器实际输出力。而作动器输出的实际电 磁力即为悬架系统的主动控制力 $F_{\mathrm{a}}$ 。

悬架系统仿真模型及主动悬架控制器参数如表 2 所示, 作动器的结构参数如表 1 所示, 仿真模型 采用 B 级路面激励，车速为 $72 \mathrm{~km} / \mathrm{h}$ 。

表 2 系统模型及控制器参数

\begin{tabular}{cc}
\hline \multicolumn{1}{c}{ 参数 } & 数值 \\
\hline 簧载质量 $M_{\mathrm{s}} / \mathrm{kg}$ & 310 \\
非簧载质量 $M_{\mathrm{u}} / \mathrm{kg}$ & 70 \\
悬架刚度 $K_{\mathrm{s}} /(\mathrm{N} / \mathrm{m})$ & 27358 \\
悬架阻尼 $C_{\mathrm{s}} /(\mathrm{N} /(\mathrm{m} / \mathrm{s}))$ & 984 \\
轮胎刚度 $K_{\mathrm{t}} /(\mathrm{N} / \mathrm{m})$ & 309511 \\
控制器参数 $q_{1}$ & $1 \times 10^{5}$ \\
控制器参数 $q_{2}$ & $1 \times 10^{6}$ \\
控制器参数 $q_{3}$ & $1 \times 10^{10}$ \\
控制器参数 $r$ & 0.01 \\
\hline
\end{tabular}

4.2 作动器输出特性分析

如图 9 所示为模型电磁力输出对比曲线, 可以 看出实际控制力与理论控制力的差异较明显, 且由 于峰值电流的限制，最大输出力有所降低，由图 10 看出, 实际电磁力的输出呈现较大的波动。

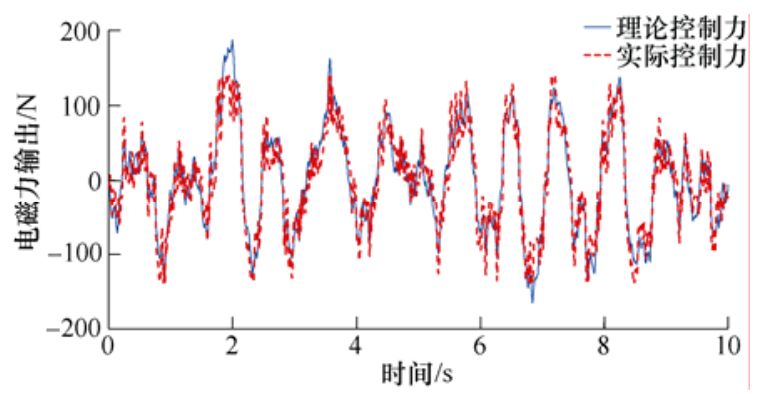

图 9 作动器电磁输出力对比

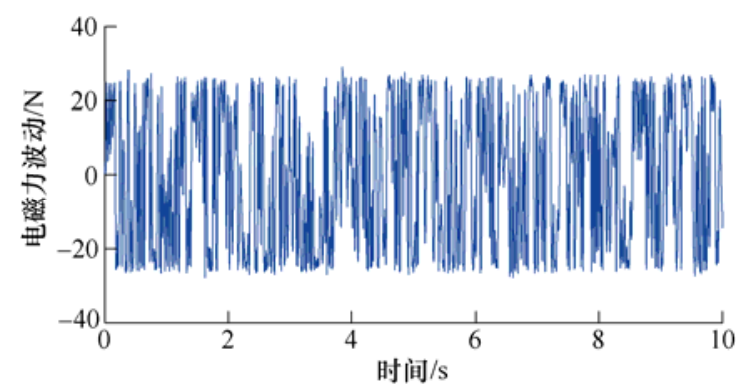

图 10 实际电磁力输出波动

表 3 所示为电磁力输出对比, 可以看出主动悬 架中的实际控制力波动值较大, 最大达到了 $28.9 \mathrm{~N}$, 而由于峰值电流的限制实际控制力的最大值及均方 根值都有一定程度的降低。 
表 3 电磁力输出对比

\begin{tabular}{cccc}
\hline 参数及单位 & 理论控制力 & 实际控制力 & 控制力波动 \\
\hline 方均根值 $/ \mathrm{N}$ & 69.5 & 65.5 & 17.8 \\
最大值 $/ \mathrm{N}$ & 188.5 & 134.2 & 28.9 \\
\hline
\end{tabular}

\section{3 悬架系统响应分析}

从作动器的输出特性可以看出, 悬架系统中作 动器实际输出的控制力较理论控制力有一定程度的 降低, 且伴随着较大的电磁力波动, 这种现象势必 会对主动悬架产生一定的影响。

图 11 为悬架系统输出的时域响应图, 可以看出 电磁力波动对悬架动挠度的影响较明显, 而对簧载 质量振动加速度及轮胎跳动的影响较小, 只在各振 动峰的峰值有一定程度的增加。通过对比表 4 , 与 时域响应曲线对应，簧载质量振动加速度、悬架动 挠度、轮胎跳动分别上升 $5.16 \% 、 4.76 \% 、 4.55 \%$ 。 图 12 为悬架系统的频响曲线对比, 可以看出电磁力 波动对悬架系统的影响在低频处较大, 各项输出频 响在一阶共振峰处都有一定程度的升高。总体来说 作动器电磁力波动会对悬架系统产生一定的性能影 响，须采取相应的措施消除这种影响。

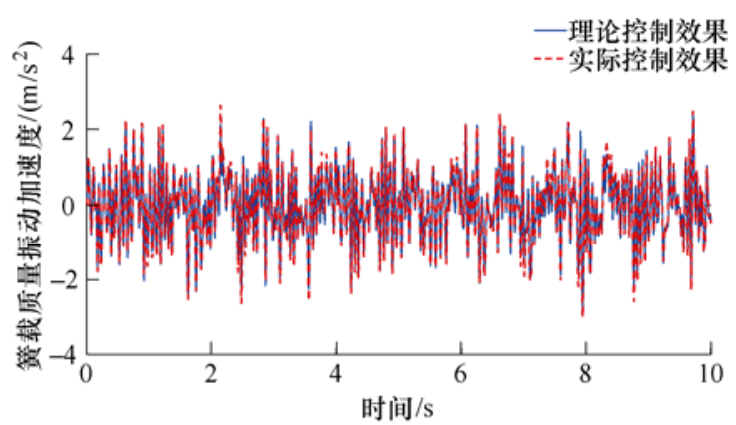

(a) 簧载质量振动加速度时域响应

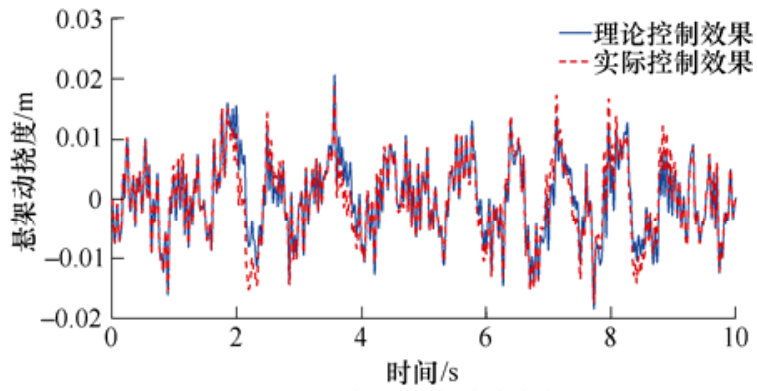

(b) 悬架动挠度时域响应

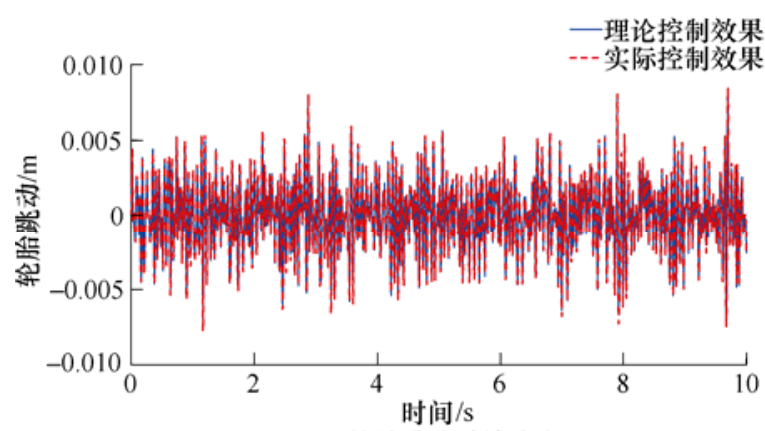

(c) 轮胎跳动时域响应

图 11 悬架系统时域响应曲线
表 4 时域响应方均根值对比

\begin{tabular}{cccc}
\hline 参数及单位 & $\begin{array}{c}\text { 理论控制 } \\
\text { 效果 }\end{array}$ & $\begin{array}{c}\text { 实际控制 } \\
\text { 效果 }\end{array}$ & $\begin{array}{c}\text { 改变 } \\
\text { 幅度 }(\%)\end{array}$ \\
\hline 簧载质量振动加速度 $/\left(\mathrm{m} / \mathrm{s}^{2}\right)$ & 0.8675 & 0.9123 & $5.16 \uparrow$ \\
悬架动挠度 $/ \mathrm{m}$ & 0.0063 & 0.0066 & $4.76 \uparrow$ \\
轮胎跳动 $/ \mathrm{m}$ & 0.0022 & 0.0023 & $4.55 \uparrow$ \\
\hline
\end{tabular}

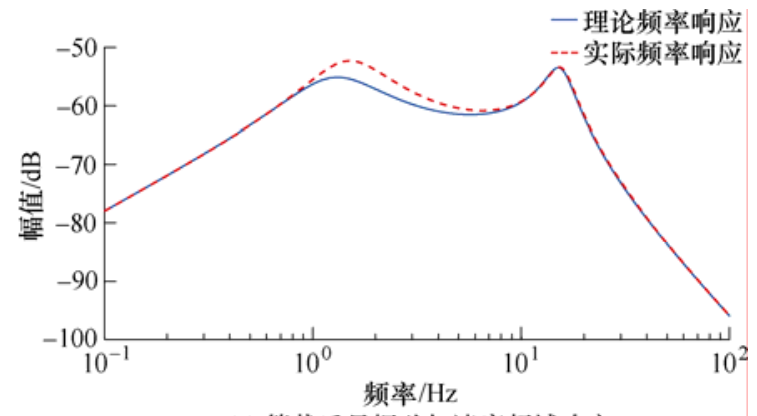

(a) 簧载质量振动加速度频域响应

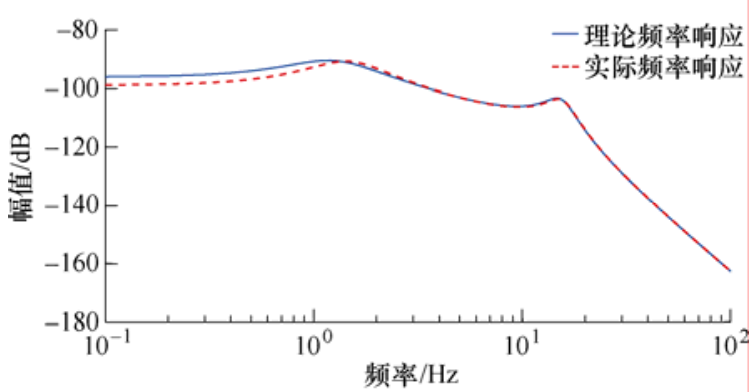

(b) 悬架动挠度频域响应

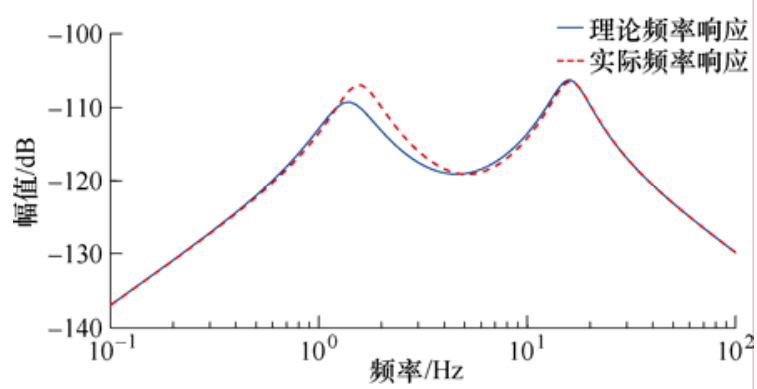

(c) 轮胎跳动频域响应

图 12 悬架系统频率响应

\section{5 基于多目标粒子群的作动器优化}

如上所述，电磁力波动会对悬架系统的动力学 性能产生一定的影响，而减小电磁力的波动，提高 有效电磁力能够减小这种影响。本文从作动器设计 的角度出发，采用基于 Pareto 支配关系的多目标粒 子群优化算法 (Multi-objective particle swarm optimization, MOPSO) 对作动器的结构参数进行优 化设计。将直线作动器优化看作为一个具有 $n$ 个优 化目标和 $u$ 个约束条件的多目标非线性优化问题， 则多目标参数优化问题描述如下 


$$
\begin{aligned}
& F_{\text {fitness }}(x)=\operatorname{best}\left\{f_{1}(x), f_{2}(x), \cdots f_{n}(x)\right\} \quad x \in T \\
T= & \left\{x \in R^{m}, G: g_{i \min } \leqslant g_{i}(x) \leqslant g_{i \max }, i=1, \cdots, u\right\}
\end{aligned}
$$

式中, $f_{1}(x), f_{2}(x), \cdots, f_{n}(x)$ 分别为第 1 到 $n$ 个优化 设计目标; $T$ 为约束条件, $R^{m}$ 为设计变量域; $G$ 为 变量约束 $; n$ 为设计目标维数; $m$ 为设计变量维数, $u$ 为约束个数。

\section{1 设计目标}

本文对作动器结构参数的优化, 一方面需要降 低感应电动势中 THD 值, 以降低电磁力的波动; 另 一方面需要提高感应电动势的幅值 $E_{\mathrm{m}}$, 以提高有效 电磁力的输出, 因此本文的设计目标维数 $n=2$ 。选 取设计目标函数如下。

目标函数 1

$$
f_{1}(x)=E_{\mathrm{m}}
$$

目标函数 2

$$
f_{2}(x)=\text { THD }
$$

为满足作动器大电磁力输出, 同时降低电磁力 中的波动，在优化过程以“大 $E_{\mathrm{m}}$, 小 $\mathrm{THD}$ ”为原 则进行优化算法中粒子的取舍。

\section{2 设计变量及约束}

根据作动器结构参数对感应电动势 THD 值和 幅值 $E_{\mathrm{m}}$ 的分析, 这里选取永磁体极弧系数 $\alpha_{\mathrm{p}}$ 、槽 宽 $\tau_{\mathrm{s}}$ 、永磁体厚度 $h_{\mathrm{m}}$ 、气隙 $g$ 为设计变量, 由初级 和次级运动不干涉、齿部强度和避免磁场饱和为要 求, 确定变量 $\alpha_{\mathrm{p}} 、 \tau_{\mathrm{s}} 、 h_{\mathrm{m}}$ 和 $g$ 的取值范围。

设计变量及约束条件如下。

设计变量为

$$
x=\left[a_{p}, \tau_{s}, h_{m}, g\right]
$$

约束条件为

$$
\begin{array}{cc}
a_{p} \in[0.3,0.8] & \tau_{S} \in[4.0,10.0] \\
h_{m} \in[2.5,5.0] & g \in[1.5,2.0]
\end{array}
$$

如上所述, 本文设计变量维数 $m=4$ 。

在形式上设计变量为连续值, 但考虑加工精度 要求, 则在优化过程中取 $0.1 \mathrm{~mm}$, 以满足作动器加 工需求。

\section{3 多目标粒子群优化算法}

在对作动器进行多目标优化时, 为避免出现陷 入局部最优以及多目标优化分配中各目标彼此冲突 等问题, 采用多目标粒子群优化算法是一最佳的选 择。多目标随机粒子群算法是基于乌群捕食行为提 出的智能优化算法, 约束处理是优化问题的一个重 要内容。为了获得更多且真实的 Pareto 解, 提高算 法全局搜索能力，本文采用一种基于自适应惩罚函
数的多目标随机粒子群优化算法, 并利用线性调整 惯性权重系数。算法中，自适应惩罚函数主要由可 变乘法因子和违反约束的乘法组成

$$
p(t, x)=\sqrt{t} \sum_{t=1}^{m} \theta\left(d_{i}(x)\right) d_{i}(x)^{r\left(d_{i}(x)\right)}
$$

式中, $t$ 为迭代次数; $m$ 为约束条件个数, 惩罚由目 标函数数量级与惩罚程度确定。

算法的流程如图 13 所示。

\section{4 优化结果分析}

本文基于作动器感应电动势幅值和总谐波畸变 率, 采用多目标粒子群优化算法对作动器结构参数 进行优化分析, 优化过程中, 采用标准学习因子： $C_{1}=C_{2}=2$, 线性权重系数为 $0.4 \sim 0.9$, 最大速度 $v_{\text {max }}$ 确定与搜索空间大小有关

$$
v_{\max }=\lambda|x|_{\max }
$$

式中， $\lambda$ 为最大速度与位置限制的比例系数。

针对本文设计作动器 $\lambda$ 取 0.1 , 种群规模为 50 , 经过 200 迭代运算, 得到悬架作动器结构参数的多 目标优化 Pareto 最优解集如图 14 所示。

由图 14 中可知，Pareto 解集包含一系列非支 配解, 由图可以看出 $E_{\mathrm{m}}$ 的增大, THD 也增大, $E_{\mathrm{m}}$ 与 THD 互相矛盾, 需从解集中选取符合要求 的解，因此如何从上述解集中选取最优解显得尤 为重要。

\section{5 基于模糊集合理论的 Pareto 解选优}

传统的人工对 Pareto 解进行选取的过程中存在 较多个人主观因素，本文采用基于模糊集合理论对 Pareto 解进行选优 ${ }^{[17]}$, 模糊集理论采用最优解方案 折中的方式并基于模糊机制帮助决策者对 Pareto 解 进行有效的选取。定义隶属函数 $S_{i}^{j}$

$$
S_{i}^{j}= \begin{cases}1 & f_{i}^{j} \leqslant f_{i}^{\min } \\ \frac{f_{i}^{\max }-f_{i}^{j}}{f_{i}^{\max }-f_{i}^{\min }} & f_{i}^{\min } \leqslant f_{i}^{j}<f_{i}^{\max } \\ 0 & f_{i}^{j} \geqslant f_{i}^{\max }\end{cases}
$$

式中, $f_{i}^{\max } 、 f_{i}^{\min }$ 分别为解集中第 $i$ 个优化目标的 最大值、最小值。 $f_{i}^{j}, S_{i}^{j}$ 为第 $j$ 个解的第 $i$ 个优 化目标的当前值与隶属度值。

定义支配函数 $\varphi_{k}$ ，第 $k$ 个解的支配值为

$$
\varphi_{k}=\sum_{i=1}^{n} s_{i}^{k} /\left(\sum_{j=1}^{l} \sum_{i=1}^{n} s_{i}{ }^{j}\right)
$$

式中,$l$ 为外部集中解的数目 根据模型的仿真结果, $l$ 取 $14 ; n$ 为悬架优化设计目标个数, 如上文所述 $n$ 取 2。 


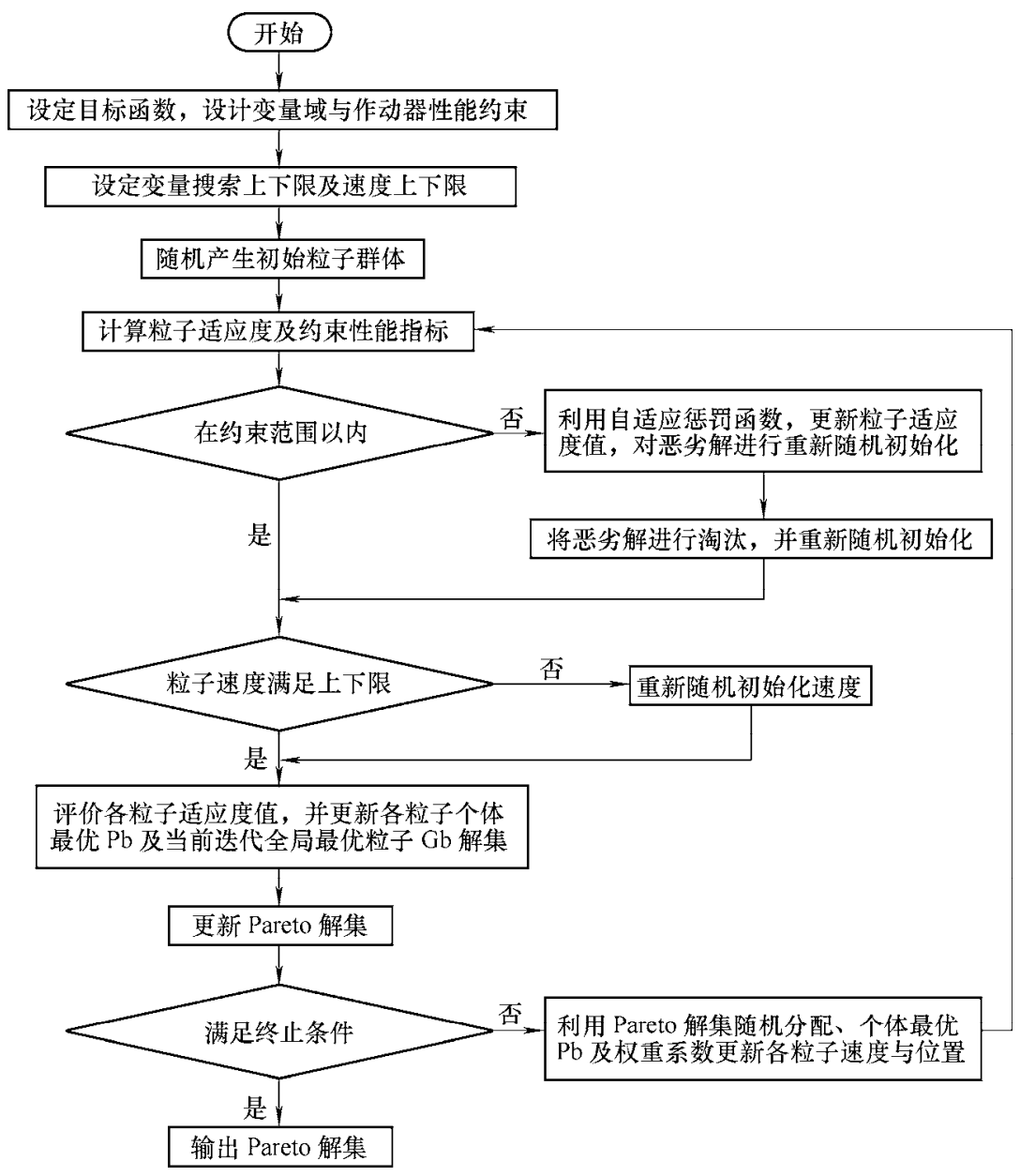

图 13 MSPSO 算法流程图

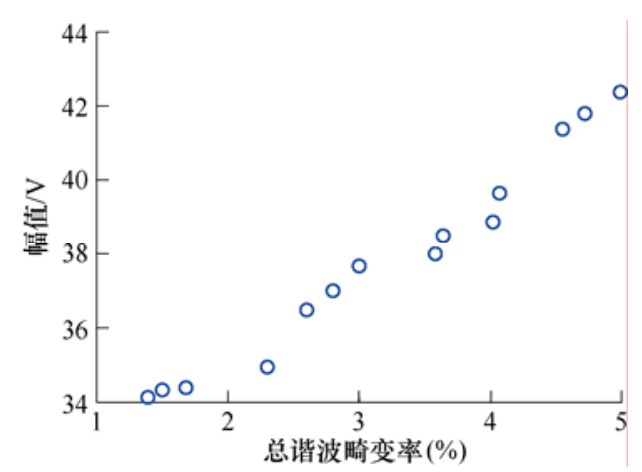

图 14 Pareto 最优解

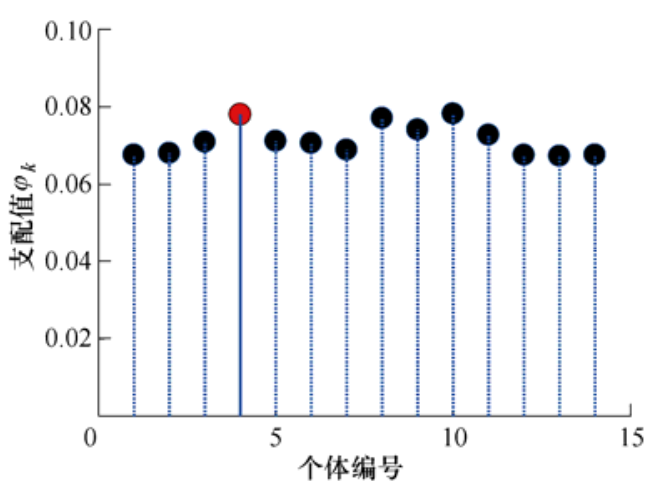

图 15 Pareto 最优解集的支配函数值

由支配函数 $\varphi_{k}$ 计算公式, 可得 Pareto 解集中 每个非劣解的支配值，通过支配值反应该解的综合 性能，选择具有较大支配值的解为最优解。如图 15 所示, 第 4 号粒子个体具有最大支配函数值, 对应作动器设计变量值及其优化前后数值如表 5 所示。

表 5 优化前后设计变量

\begin{tabular}{lcc}
\hline 设计变量及单位 & 优化前 & 优化后 \\
\hline 永磁体厚度 $h_{\mathrm{m}} / \mathrm{mm}$ & 3.0 & 4.5 \\
气隙长度 $\mathrm{g} / \mathrm{mm}$ & 2.0 & 1.7 \\
极弧系数 $\alpha_{\mathrm{p}} / \mathrm{mm}$ & 0.8 & 0.6 \\
齿宽 $\tau_{\mathrm{r}} / \mathrm{mm}$ & 7.0 & 6.0 \\
\hline
\end{tabular}

\section{6 优化结果分析}

根据表 3 所得作动器优化前后结构参数, 对优 化前后作动器电磁力进行对比分析。图 16 为在运行 速度为 $0.24 \mathrm{~m} / \mathrm{s}$ 时, 作动器优化前后绕组感应电动 势对比曲线, 图 17 为在幅值为 $2 \mathrm{~A}$ 的交流电激励下, 优化前后作动器电磁力曲线。优化前后作动器具体 性能参数对比如表 6 所示。 


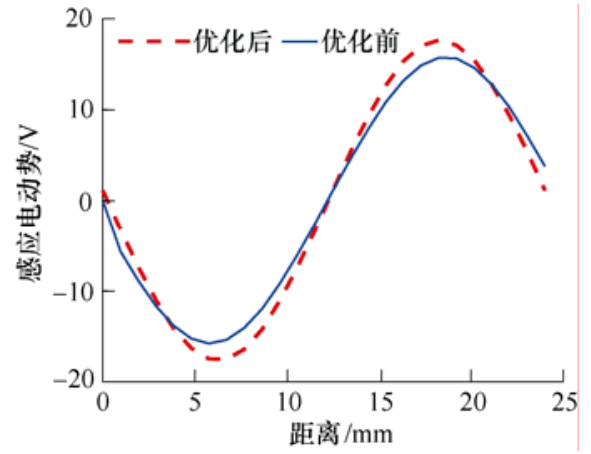

图 16 感应电动势曲线对比图

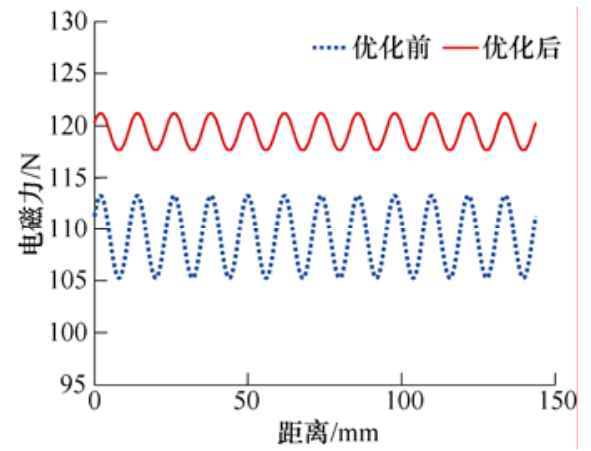

图 17 电磁力曲线对比图

表 6 优化前后作动器关键参数结果对比

\begin{tabular}{lccc}
\hline \multicolumn{1}{c}{ 参数及单位 } & 优化前 & 优化后 & 改变幅度 $(\%)$ \\
\hline 感应电动势幅值 $E_{\mathrm{m}} / \mathrm{V}$ & 15.2 & 16.8 & $10.5 \uparrow$ \\
$\mathrm{THD}$ 值 $(\%)$ & 5.6 & 2.7 & $51.8 \downarrow$ \\
电磁力波动值 $F_{\mathrm{r}} / \mathrm{N}$ & 7.8 & 3.6 & $53.8 \downarrow$ \\
电磁力 $F / \mathrm{N}$ & 109.2 & 118.5 & $8.5 \uparrow$ \\
\hline
\end{tabular}

从图 16 可以看出 : 在定工况下, 经过优化后感 应电动势较优化前作动器感应电动势幅值有一定的 升高, 波形较优化前也有一定变化; 图 17 中可以看 出: 优化后的电磁力整体波动幅度较优化前有了降 低, 同时平均值也得到提高。优化前后作动器关键 参数结果对比如表 6 所示。

表 6 所示优化前作动器工作速度在 $0.24 \mathrm{~m} / \mathrm{s}$ 时 感应电动势幅值约为 $15.2 \mathrm{~V}$, 优化后上升为 $16.8 \mathrm{~V}$, 增大了 $1.6 \mathrm{~V}$ ，提高了约 $10.5 \%$; 感应电动势 THD 值由原来的 $5.6 \%$ 下降为 $2.7 \%$ ，降低了约 $51.8 \%$; 电 磁力波动值在 $2 \mathrm{~A}$ 时由 $7.8 \mathrm{~N}$ 下降为 $3.6 \mathrm{~N}$, 下降了 约 53.8\% 电磁力由优化前的 $109.3 \mathrm{~N}$ 增加到了 118.5 $\mathrm{N}$ ，提高约 $8.5 \%$ 。可以看出，采用多目标粒子群优 化方法对本文作动器的优化, 提高了感应电动势和 电磁力, 同时显著降低了感应电动势 THD 值和电磁 力的波动。

\section{7 优化后主动悬架特性分析}

将优化后的结构参数代入主动悬架系统仿真 模型中进行仿真, 如图 18 所示为优化前后电磁力 输出对比曲线, 可以看出优化后的实际电磁力输
出峰值相对优化前有明显的提升，而从图 19 可以 看出实际电磁力的波动值也较优化前有了明显的 降低。

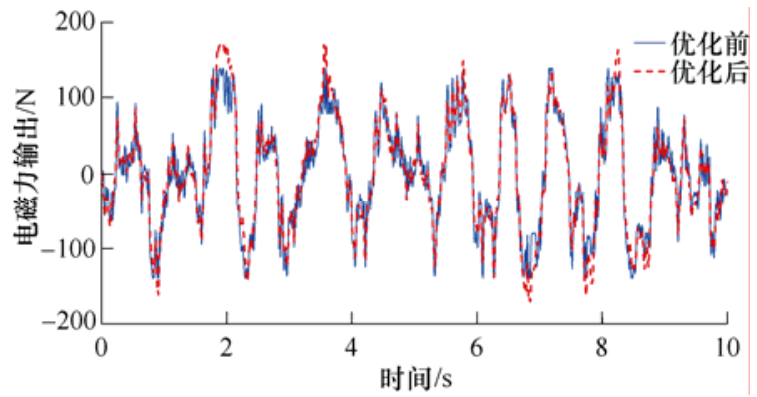

图 18 作动器电磁输出力对比

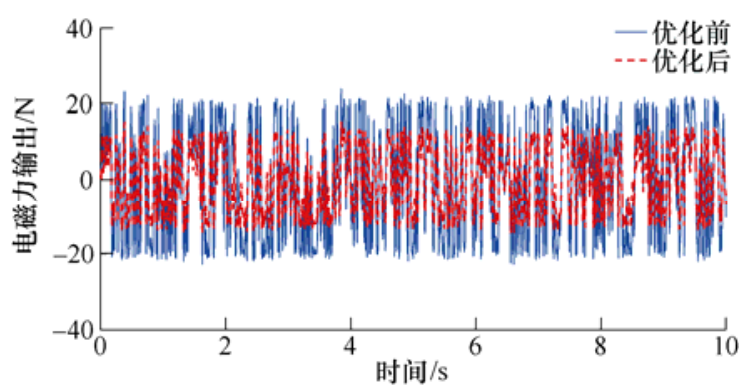

图 19 实际电磁力输出波动

由表 7 可以看出实际电磁力输出方均根值与最 大值分别上升 $4.5 \%$ 与 $21.3 \%$, 电磁力波动方均根值 与最大值分别下降 $51.7 \%$ 与 $45 \%$ 。

表 7 电磁力输出对比

\begin{tabular}{lccc}
\hline \multicolumn{1}{c}{ 参数及单位 } & 优化前 & 优化后 & 改变幅度 $(\%)$ \\
\hline 电磁力方均根值 $/ \mathrm{N}$ & 65.5 & 68.6 & $4.5 \uparrow$ \\
电磁力最大值 $/ \mathrm{N}$ & 134.2 & 170.5 & $21.3 \uparrow$ \\
电磁力波动方均根值 $/ \mathrm{N}$ & 17.8 & 8.6 & $51.7 \downarrow$ \\
电磁力波动最大值 $/ \mathrm{N}$ & 28.9 & 15.9 & $45 \downarrow$ \\
\hline
\end{tabular}

图 20 所示为优化前后的系统输出时域响应对 比曲线，可以看出优化后的系统响应都有小幅降低， 而通过表 8 的时域响应方均根值对比也能看出，优 化后的系统各项动力学响应都有小幅的降低，基本 接近悬架系统的理论控制效果。而从图 21 系统输出 的频响曲线也能看出，优化后系统在低频处响应恶 化的特性得到了一定程度的改善。

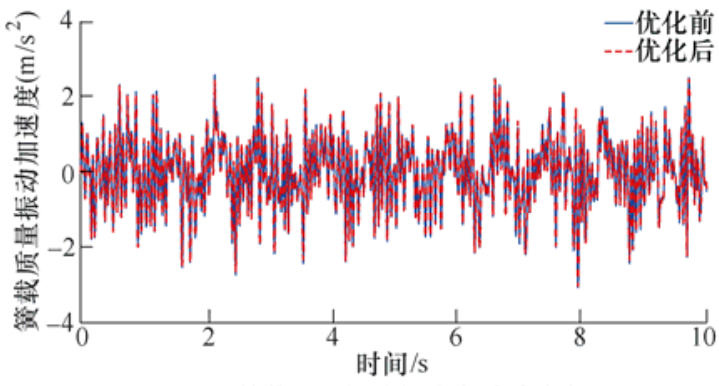

(a) 簧载质量振动加速度时域响应 


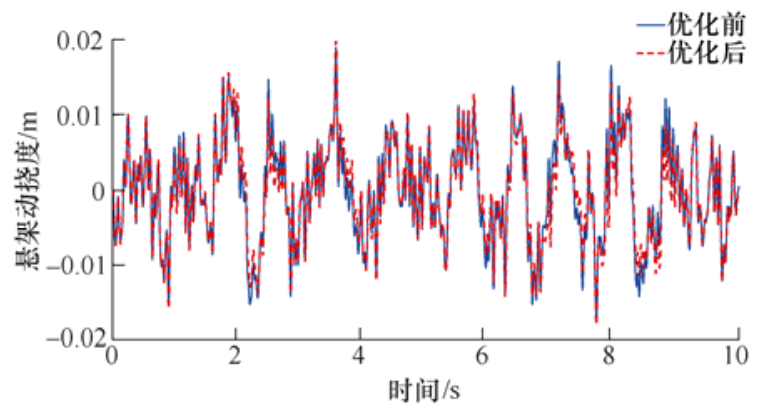

(b) 悬架动挠度时域响应

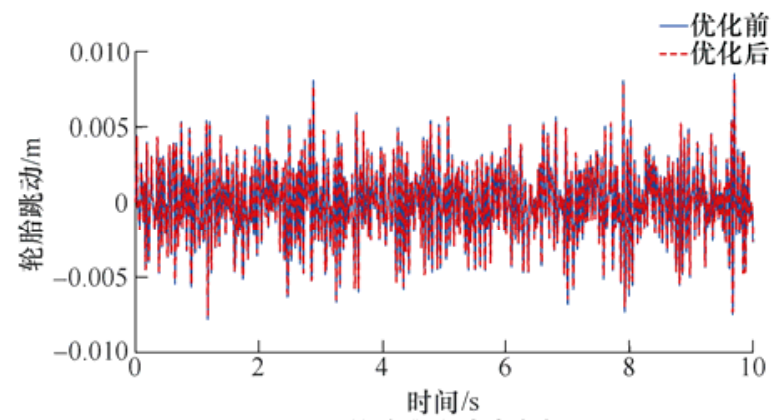

(c) 轮胎跳动时域响应

图 20 优化前后悬架系统时域响应

表 8 时域响应方均根值对比

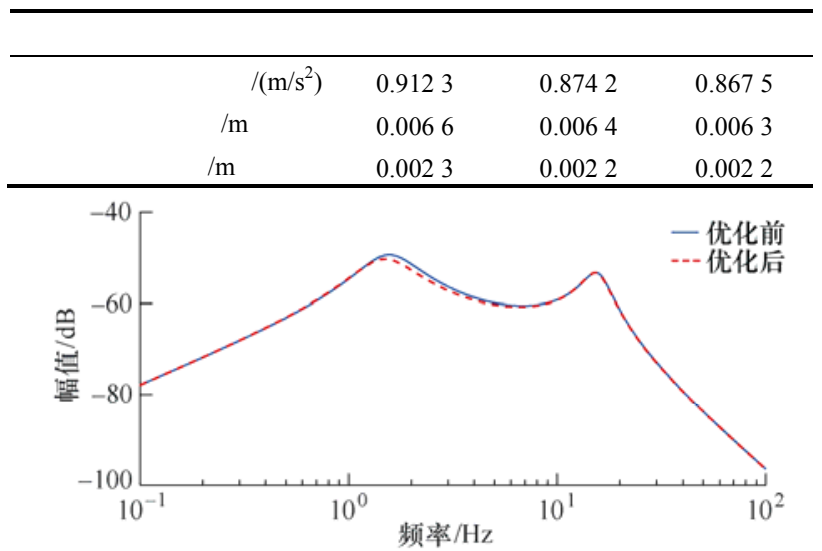

(a) 簧载质量振动加速度频域响应

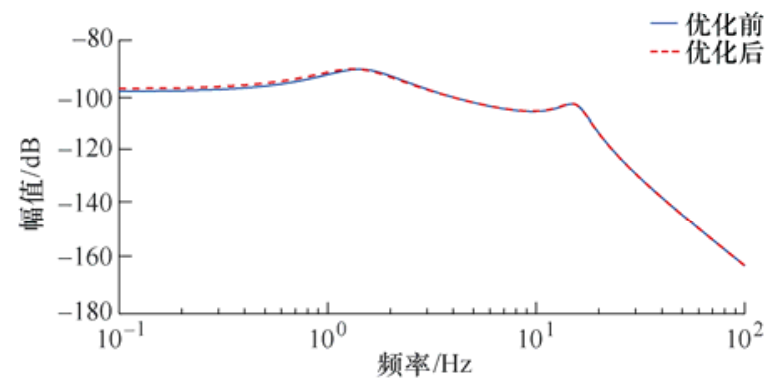

(b) 悬架动挠度频域响应

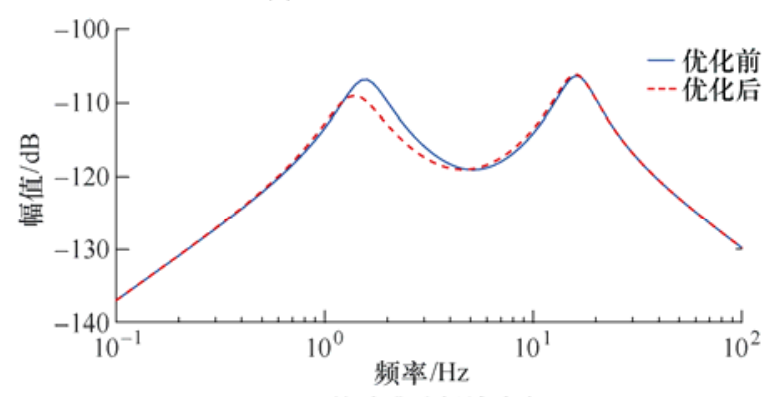

(c) 轮胎跳动频域响应

图 21 优化前后悬架系统频率响应

\section{6 试验}

根据作动器的设计及优化后的结构尺寸, 加 工制作了一套作动器样件如图 22 所示。对样件性 能参数进行测试, 以验证上述作动器结构优化的 结果。

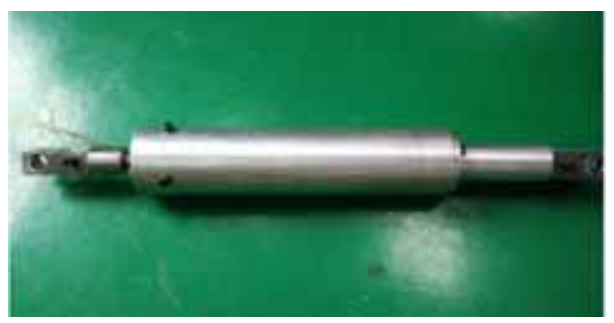

图 22 作动器样件图

针对作动器的测试, 这里借助减振器测试试验 台, 依据 QC/T545-1999 汽车筒式减振器台架试验 方法, 开展作动器电磁力及其波动的测试。作动器 测试设备主要包括 WDTS-IV 减振器综合电测示功 机、计算机、夹具、电磁悬架作动器等。主要技术 参数: 最大激振力为 $20 \mathrm{kN}$, 最大激振幅度为 \pm 80 $\mathrm{mm}$, 简谐振动的最大速度为 $1.2 \mathrm{~m} / \mathrm{s}$, 最大加速度 为 $20 \mathrm{~g}$ 。力传感器的量程为 $-10 \sim 10 \mathrm{kN}$ 。感应电压 的测试采用 SDS1102CN 数字示波器进行显示。电 磁力的测试, 可以通过在外部设置可调盘式负载电 阻，测试台架如图 23 所示。

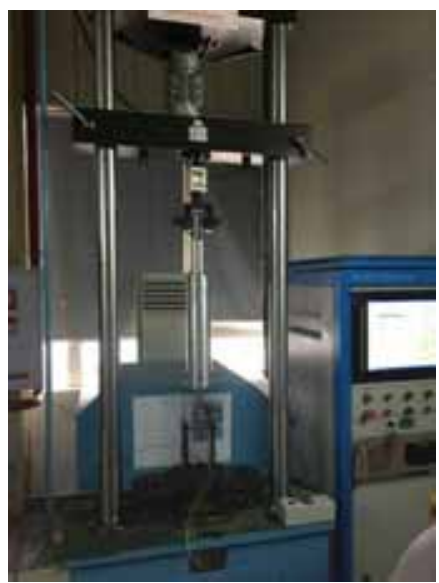

图 23 作动器台架测试

电磁力测试前, 将样件垂直安装在试验台上, 如图 23 所示, 以工作行程约二分之一处为试验行 程中点, 分别以最大速度 $0.1 \mathrm{~m} / \mathrm{s}, 0.2 \mathrm{~m} / \mathrm{s}$ 和 0.3 $\mathrm{m} / \mathrm{s}$ 在 $\pm 25 \mathrm{~mm}$ 行程的激励下加振, 往复运动 1 次 采样，绘制一个周期的示功图，如图 24 所示，其 中外部可调电阻设为 $1 \Omega$ 。表 9 所示为测试值和 试验值对比。 


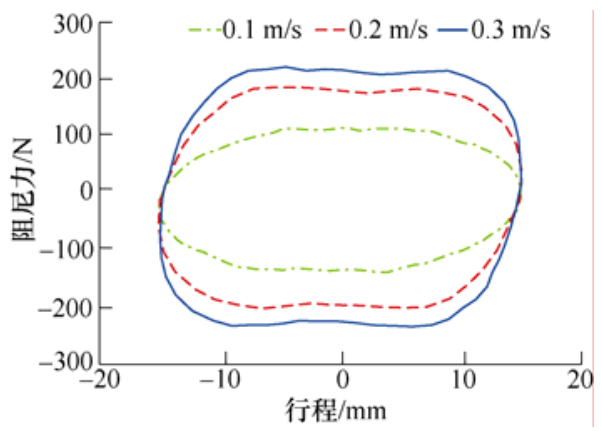

图 24 作动器电磁力

表 9 电磁力波动值试验值和仿真值对比

\begin{tabular}{cccc}
\hline & 速度 $/(\mathrm{m} / \mathrm{s})$ & 仿真 $/ \mathrm{N}$ & 试验 $/ \mathrm{N}$ \\
\hline \multirow{3}{*}{ 波动差 } & 0.1 & 3.4 & 11.6 \\
& 0.2 & 5.8 & 14.3 \\
& 0.3 & 6.3 & 14.9 \\
\hline
\end{tabular}

从上述测试可以看出，优化后的电磁力波动的 仿真值在对应速度 $0.1 \mathrm{~m} / \mathrm{s}, 0.2 \mathrm{~m} / \mathrm{s}$ 和 $0.3 \mathrm{~m} / \mathrm{s}$ 时分 别为 $3.4 \mathrm{~N}, 5.8 \mathrm{~N}$ 和 $6.3 \mathrm{~N}$, 而试验值分别为 $11.6 \mathrm{~N}$, $14.3 \mathrm{~N}$ 和 $14.9 \mathrm{~N}$, 试验值明显比仿真值大, 主要是 因为测试中, 电磁力的波动存在定位力的影响, 即 作动器仍存在部分定位力 (主要和作动器长度相 关)。为证明本文优化的有效性, 需要对定位力进行 测试并剔除。而对定位力的测试，这里通过静摩擦 力测试的方式来实现。以 $0.005 \mathrm{~m} / \mathrm{s}$ 作为试验速度 (电压极小, 默认作动器电流为 0 ), 往复运动 1 次 采样, 记录一个周期的静摩擦力值。测试结果如图 25 所示。

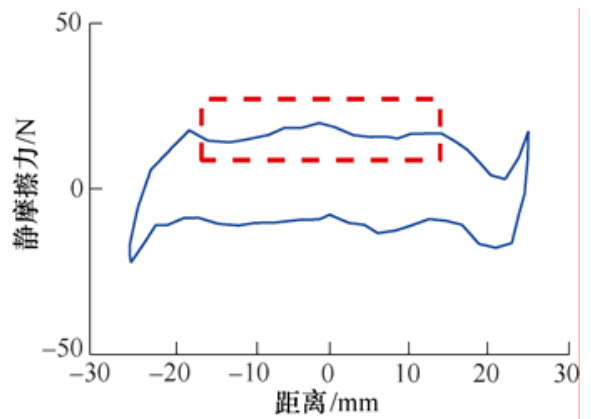

图 25 静摩擦力特性曲线

从图 25 中可以看出, 虚线框区域所显示部分静 摩擦力呈现出一定的波动, 此部分的波动差即为定 位力。故采用虚线框区域内的最大值和最小值之差 来预估定位力。通过计算发现本文作动器的定位力 值约为 $7.8 \mathrm{~N}$ 。

基于定位力的测试, 以及表中的分析，在不考 虑定位力的基础上，对比优化后电磁力波动值的仿 真分析结果和测试结果, 如表 10 所示。可以看出优 化后在不考虑定位力的基础上, 电磁力波动值与测 试值相差约为 $10.3 \% \sim 12.7 \%$, 相差较小, 证明了本
文关于作动器电磁力波动优化的有效性。

表 10 电磁力波动值试验值和仿真值对比

\begin{tabular}{ccccc}
\hline & 速度 $/(\mathrm{m} / \mathrm{s})$ & 仿真 $/ \mathrm{N}$ & 试验 $/ \mathrm{N}$ & 差值 $(\%)$ \\
\hline \multirow{4}{*}{ 波动差 } & 0.1 & 3.4 & 3.8 & 11.7 \\
& 0.2 & 5.8 & 6.4 & 10.3 \\
& 0.3 & 6.3 & 7.1 & 12.7 \\
\hline
\end{tabular}

针对感应电压的测试, 分别以最大速度 0.1 $\mathrm{m} / \mathrm{s}, 0.2 \mathrm{~m} / \mathrm{s}$ 和 $0.3 \mathrm{~m} / \mathrm{s}$ 在 $\pm 50 \mathrm{~mm}$ 行程的激励下加 振, 每个速度下往复运动 $5 \sim 8$ 次采样, 截取各速度 激励下的一个周期的三相感应电压，结果如图 26 所示。

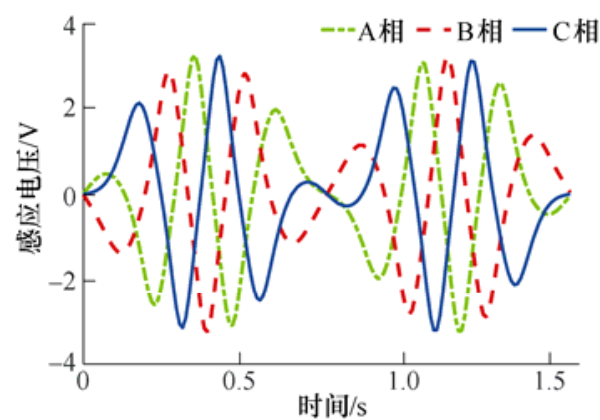

(a) $0.1 \mathrm{~m} / \mathrm{s}$ 感应电压曲线

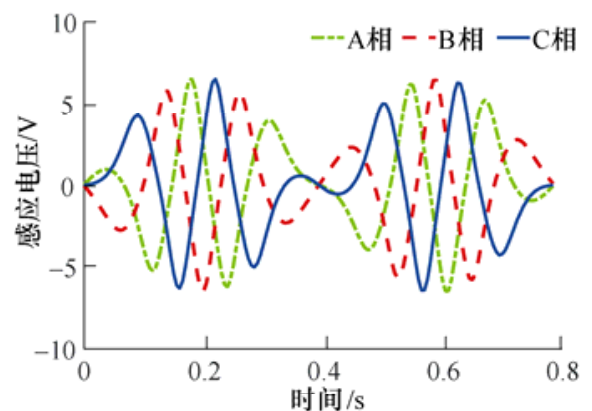

(b) $0.2 \mathrm{~m} / \mathrm{s}$ 感应电压曲线

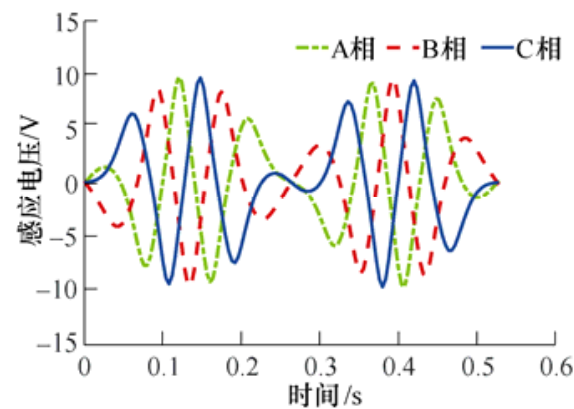

(c) $0.3 \mathrm{~m} / \mathrm{s}$ 感应电压曲线

图 26 不同速度下感应电压曲线

由图 26 可知，随着试验台激振速度的增加，作 动器感应电压逐渐增加，但周期变短。 $0.1 \mathrm{~m} / \mathrm{s}, 0.2$ $\mathrm{m} / \mathrm{s}$ 和 $0.3 \mathrm{~m} / \mathrm{s}$ 对应的感应电压的幅值的平均值分别 为 $3.1 \mathrm{~V}, 6.4 \mathrm{~V}$ 和 $9.5 \mathrm{~V}$, 基本呈线性增加。由于 $\mathrm{A}$ 相、 $\mathrm{B}$ 相和 $\mathrm{C}$ 相互成 $120^{\circ}$ 夹角, 故作动器三相感应 电压曲线并不相同，但幅值基本相同。 


\section{7 结论}

针对直线式电磁悬架作动器电磁力波动对主动 悬架的影响问题, 建立电磁力波动的解析模型, 在 此基础上, 建立考虑电磁力波动的主动悬架动力学 模型，分析了电磁力波动对主动悬架的影响，借助 多目标粒子群智能优化算法, 对作动器本体结构参 数进行优化设计, 在降低了电磁力波动的同时提高 了有效电磁力的输出, 基本消除了作动器电磁力波 动对主动悬架性能的影响。并通过试验验证了作动 器本体优化设计的有效性。所得结论如下。

(1) 当输入 $2 \mathrm{~A}$ 三相正弦交流电时, 作动器电磁 力波动高达 $7.8 \mathrm{~N}$; 在感应电动势谐波分析中发现： 除基波分量外还存在 3 次、 2 次、4 次和 5 次等谐波 分量, 总谐波畸变率 THD 值约为 $5.6 \%$ 。感应电动 势中的谐波造成了电磁力的波动。

(2) 考虑电磁力波动时, 电磁主动悬架系统簧 载质量振动加速度、悬架动挠度以及轮胎跳动响应 分别增加 $5.16 \% 、 4.76 \% 、 4.55 \%$ ，对系统动力学性 能产生了一定的影响。实际工况下作动器电磁力波 动高达 $28.9 \mathrm{~N}$ 。

(3) 在作动器结构参数对感应电动势影响的分 析中发现: 作动器齿宽、永磁体厚度、极弧系数和 气隙长度对感应电动势幅值和波形具有较大影响。 运用多目标粒子群智能优化算法对作动器结构参数 进行了优化, 结果显示: 定工况下优化后感应电动 势幅值提高了约 $10.5 \%$,总谐波畸变率 THD 值降低 了约 $51.8 \%$; 电磁力波动值下降了约 $53.8 \%$, 电磁 力输出值提高约 $8.5 \%$ 。实际工况下，作动器输出的 实际电磁力波动降低 $45 \%$, 电磁力波动方均根值下 降 51.7\%，极大的降低了电磁力的波动特性对悬架 系统性能的影响，优化后的系统响应接近理想控制 情况。

(4) 在不考虑定位力的情况下, 优化后作动器 电磁力波动值的定工况下的仿真值与测试值较为接 近, 相差仅为 $10.3 \% \sim 12.7 \%$, 从而验证了本文关于 作动器电磁力波动优化的有效性, 能够为后续类似 作动器优化提供参考。

\section{参 考 文 献}

[1] MURATTA S. Innovation by in-wheel-motor drive unit [J]. Vehicle System Dynamics , 2012 , 50(6) : 807-830.

[2] WANG Yanyang, LI Yinong ,SUN Wei ,et al. Effect of the unbalanced vertical force of a switched reluctance motor on the stability and the comfort of an in-wheel motor electric vehicle $[\mathrm{J}]$. Proc IMechE Part D : Journal of Automobile Engineering , 2015 , 229(12) : 1569-1584.

[3] KANGHYU N ,FUJIMOTO H ,HORI Y. Lateral stability control of in-wheel-motor-driven electric vehicles based on sideslip angle estimation using lateral tire force sensors [J]. IEEE Transactions on Vehicular Technology , 2012 , 61(5) : 1972-1985.

[4] THUL A , EGGERS D , RIEMER B , et al. Active suspension system with integrated electrical tubular linear motor : Design, control strategy and validation [J]. Archives of Electrical Engineering ,2016,64(4):605-616.

[5] WANG Wei, SONG Yulin, XUE Yanbing, et al. An optimal vibration control strategy for a vehicle's active suspension based on improved cultural algorithm [J]. Applied Soft Computing , 2015 , 28 : 167-174.

[6] 裴金顺，李以农，王艳阳，等. 采用电磁直线电机的主 动悬架控制系统的设计与能量分析 [J]. 汽车工程, 2014, 36(11) : 1386-1391.

PEI Jinshun , LI Yinong, WANG Yanyang, et al. Design and energy analysis of an active suspension control system with electromagnetic linear motor $[\mathrm{J}]$. Automotive Engineering , 2014 , 36(11) : 1386-1391.

[7] ZHANG Guogang, CAO Jianyong, YU Fan. Design of active and energy-regenerative controllers for $\mathrm{dc}$ motor-based suspension [J]. Mechatronics , 2012 , 22(8) : 1124-1134.

[8] HUANG Bo , HSIEH Chenyu , GOLNARAGHI F , et al. Development and optimization of an energy-regenerative suspension system under stochastic road excitation [J]. Journal of Sound and Vibration , 2015 , 357 : 16-34.

[9] 邓兆祥, 来飞. 车辆主动悬架用电磁直线作动器的研究 [J]. 机械工程学报, 2011，47(14)：121-128.

DENG Zhaoxiang, LAI Fei. Electromagnetic linear actuator for vehicle active suspension $[\mathrm{J}]$. Journal of Mechanical Engineering , 2011 , 47 (14) : 121-128.

[10] LIN Jiongkang, CHENG K W E , ZHANG Zhu , et a1. Active suspension system based on linear switched reluctance actuator and control schemes [J]. IEEE Transactions on Vehicular Technology , 2013, 62(2) : $562-572$.

[11] 杨超，李以农，钟银辉，等. 一种分数槽结构的悬架直 线作动器及性能分析 $[\mathrm{J}]$. 上海交通大学学报, 2016 , 50(6) : 837-843.

YANG Chao ,LI Yinong ,ZHONG Yinhui ,et al. A tubular permanent magnet linear actuator based on fractional slot and performance analysis [J]. Journal of Shanghai Jiaotong University , 2016 , 50(6) : 837-843.

[12] BART L J G , JEROEN L G J , JOHANNES J H P , et al. Design aspects of an active electromagnetic suspension 
system for automotive applications[J]. IEEE Transactions on Industry Applications , 2010 , 45(5) : 1589-1597.

[13] WANG Qian , WANG Jianbin. Assessment of cogging-force-reduction techniques applied to fractional-slot linear permanent magnet motors equipped with non-overlapping windings [J]. LET Electric Power Applications , $2016,10(8):$ 697-705.

[14] WANG Jianbin , DAVID H. Tubular modular permanent-magnet machines equipped with quasi-halbach magnetized magnets - part i : Magnetic field distribution , emf , and thrust force [J]. IEEE Transactions on Magnetics , 2005 , 41(9) : 2470-2478.

[15] 谭建成. 永磁无刷直流电机技术 $[\mathrm{M}]$. 北京 : 机械工业 出版社, 2011.

TAN Jiancheng. Technology of permanent magnet brushless DC motor [M]. Beijing : China Machine Press , 2011.

[16] WANG Jianbin , GERAINT W J , DAVID H. A general framework for the analysis and design of tubular liear permanent magnet machines [J]. IEEE Transactions on Magnetics , 1999 , 35(3) : 1986-2000.

[17] ABIDO M A. Multiobjective evolutionary algorithms for electric power dispatch problem[J]. IEEE Transactions on Evolutionary Computation , 2006 , 10(3) : 315-329.

作者简介: 杨超, 男, 1986 年出生, 博士研究生。主要研究方向为车辆 振动噪声控制。

E-mail : cyang@cqu.edu.cn

李以农(通信作者), 男, 1961 年出生, 博士, 教授, 博士研究生导师。 主要研究方向为车辆系统动力学与控制、振动噪声控制。

E-mail : ynli@cqu.edu.cn 